\title{
Experimental Study on Natural Fibers for Green Acoustic Absorption Materials
}

\author{
Lamyaa Abd ALRahman, Raja Ishak Raja and Roslan Abdul Rahman
}

Department of System Dynamics, Faculty of Mechanical Engineering,

University Technology Malaysia, 81310, Skudai, Johor, Malaysia

Received 2013-01-01, Revised 2013-04-18; Accepted 2013-09-05

\begin{abstract}
Natural fibre materials are one of the major ways to improve environmental pollution and new materials need to be considered to find the best solutions. This study presents an experimental investigation on pure micro porous materials from two types of fibers: Date Palm Fibre (DPF) and Coconut Coir Fibre (CCF). This study was conducted to examine the potential for using these two types of fibers as sound absorbers. To account for the effects of the characteristics of these materials, such as thickness and density, on the acoustic absorption coefficient of a sound absorber, the measurements were conducted in an impedance tube on normal incidence acoustic absorption. The experimental data indicate that two peak values of Acoustic Absorption Coefficient (AAC) are 0.98 at $1381.25 \mathrm{~Hz}-1506.25 \mathrm{~Hz}$ for the $40 \mathrm{~mm}$ thick sample and the AAC at high frequency for the same thickness is 0.99 at $4521.88-4906.25 \mathrm{~Hz}$; for $20 \mathrm{~mm}$ thickness, the peak value is 0.84 at $2606.25-3025 \mathrm{~Hz}$. The AAC of the CCF sample is 0.77 at $2434.38-2543.75 \mathrm{~Hz}$ for a $40 \mathrm{~mm}$ thickness, but for $20 \mathrm{~mm}$ thickness, the value is 0.71 at $4184.38-4575 \mathrm{~Hz}$. The Acoustic Absorption Coefficients (AAC) of the two types of materials were increased at all frequencies when the thickness of the sample was increased. The results show that date palm fibre and coconut coir fibre have good acoustic properties at low and high frequencies and can be used as an alternative replacement to conventional products. The comparisons between the two panels show a good potential because they are cheaper and lighter in comparison to asbestos and rock wool industrial materials.
\end{abstract}

Keywords: Date Palm Fibre, Coconut Coir Fibre, Acoustic Absorption Coefficient, Density, Thickness

\section{INTRODUCTION}

Natural fibre materials are increasingly being used for different purposes in many specialised applications. Along with technology development, environmental noise causes many negative effects, according to a recent statistical study that revealed that a percentage of the population was suffering from (53\%) headaches, (36\%) high blood pressure, (40\%) anxiety, (36\%) hearing disabilities, (15\%) cardiovascular diseases, (67\%) irritability and (61\%) insomnia(Gupta and Ghatak, 2011). Therefore, there are several methods for mitigating noise problems via reused sound absorption materials. However, previous methods using synthetic materials, such as glass wool, rock wool, asbestos, have disadvantages in that they are health hazards for lungs and eyes (Suter, 2002). Currently, several researchers have been investigating this issue and findings have revealed the potential of innovative natural fibers; for example, (Koizumi et al., 2002) used bamboo fibers and (Yang et al., 2003) studied the absorption coefficients of four fibre assemblies," cashmere, goose down, acrylic fibre and kapok". These materials are natural and acrylic fibers. Natural fibers have distinctive internal structures that influence the sound absorption coefficients, which are measured according to the density, thickness and sound frequency to check the contribution of natural fibre against air. Khedari et al. (2003) studied new particle board manufactured using durian peel and coconut coir fibers to achieve the lowest thermal conductivity to decrease heat transfer into a of System Dynamics, Faculty of Mechanical Engineering, University Technology Malaysia, 81310, Skudai, Johor, Malaysia 
space. In terms of heat reduction, these agriculture wastes are an economical and interesting option that could be utilised to insulate ceilings and walls. After a year, (Khedari et al., 2004) developed a particleboard of low thermal conductivity manufactured using a mixture of durian peel and coconut coir at an optimum ratio of 90:10 (coconut coir to durian) by weight. The density was $856 \mathrm{~kg} / \mathrm{m}^{3}$, the thickness was $10 \mathrm{~mm}$ and the ratio of extracted coconut coir to durian fibre was quite low, with their differences between 0.0728 and $0.1342 \mathrm{~W} / \mathrm{m} \mathrm{K}$.

Meanwhile, (Ersoy and Kucuk, 2009) investigated the sound absorption of industrial tea leaves waste developed into three different layers with or without a single backing layer of woven textile cloth to test its experimental properties of sound absorption. The data indicated that the sound absorption properties increased by increasing the thickness of the layer of single backing cotton cloth. This result means that the natural material and renewable material has positive sound attenuation properties and most importantly, it poses lesser or no harm to human health.

Mahzan et al. (2010) investigated the feasibility of composite from coconut coir, with addition second-hand tube rubber, for sound absorption material. So, the affect of adding second-hand rubber particles and sway of polyurethane are investigate, as possible substitute of industrial and metal fibers. To obtain the higher coefficient absorption sound. On the other hand, by using these components for preserve the environment from pollution and noise reduction.

Ayub et al. (2009) explored the capacity of sound absorption of natural coir fibre using the Delany-Bazley model for three coir fibre samples by increasing the thickness, corresponding to an increase in the absorption coefficient. The performance is more promising at lower frequencies. Rozli et al. (2009a) studied the sound absorption coefficient between coconut fibre and oil palm fibre. The results depend on many acoustic absorption factors, such as thickness, density and others. These natural fibre materials have many benefits; they are renewable, abundant, less expensive, less hazardous to health and lighter in weight, as well as environmentally friendly in comparison to other synthetic substances.

Hosseini et al., (2010) studied the absorption coefficients for fresh and synthetic coconut fibers mixed with a binder and the analysis was performed using the typical "Delaney-Bazley and Biot-Allard analysis". This study found that the binder additive is not sufficient to improve the absorption coefficient for lower frequencies, so to improve the sound absorption properties; the added materials must be able to improve properties such as "stiffness, fire retardant, anti-fungus and flammability".

Arenas and Crocker (2010) compared among the ancient substances and the new substances at acoustic porosity, so developed at high quality, perfect to become more safety and thinner. Moreover, these substances are more smoothly to build up environment, hence using organic substances in to decrease noise. In order for creating novel or develop these. For this reason, many researches concentrating in acoustic substances absorption may be more efficiency and activity in future.

This study investigated the potential use micro porous materials of date palm fibre and coconut fibre instead of synthetic materials for acoustic applications. This study studied the acoustic characteristics for two natural micro porous fibers using an impedance tube and two thicknesses and densities were used to determine the acoustic absorption coefficient. Diffuse field sound absorption was measured and validated in an impedance tube obtained by using SCS software. Further, analyses on the normal incidence absorption of coir fibre and date palm fibre were conducted in an acoustic laboratory.

\section{MATERIALS AND METHODS}

The acoustic absorption behaviour of a micro porous material was studied for two different thicknesses, 20 $\mathrm{mm}$ and $40 \mathrm{~mm}$, with various densities. Crude fibers are processed to make a sample for testing of the material's sound absorption coefficient. Crude fibre was compressed to make the sample using moulds. Therefore, the sample contains almost the same composition (including the granular matrix) as when it was collected as shown in Fig. 1. Samples were collected as large rectangular panels and then cut into a circular shape suitable for the impedance tube test. Fibre sample preparation steps are as follows.

\subsection{Fibre Chopping Process}

Raw micro porous materials from natural fibre were cut using a special large machine. The result is a mixture of small pieces of fibre filaments, small pieces of wood and fibre powder, which looks like powder dust.

\subsection{Fibre Extraction Process}

In this process, fibre filaments were separated from the rest of the materials. The chopped materials were filtered from impurities to isolate the fibre filaments from the rest of the material. This operation was carried out manually. 


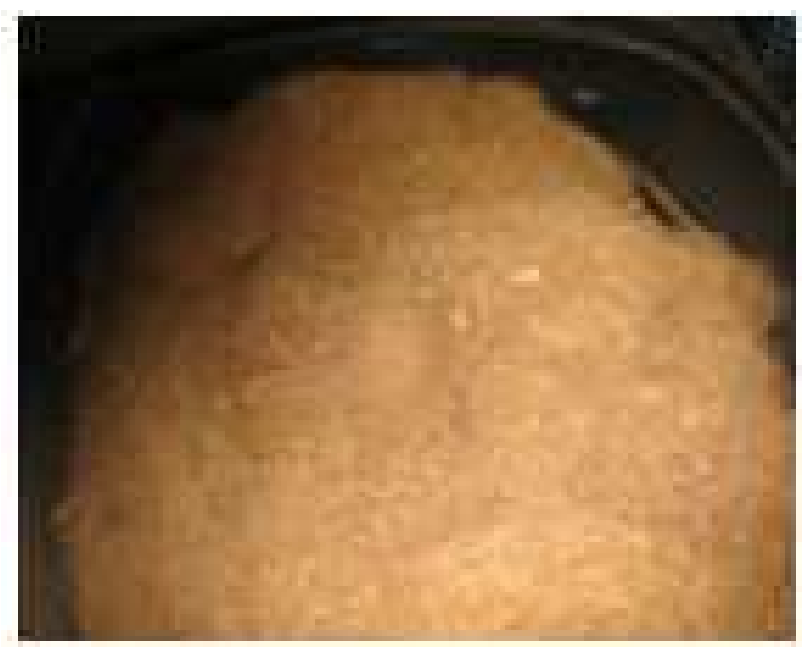

(a)

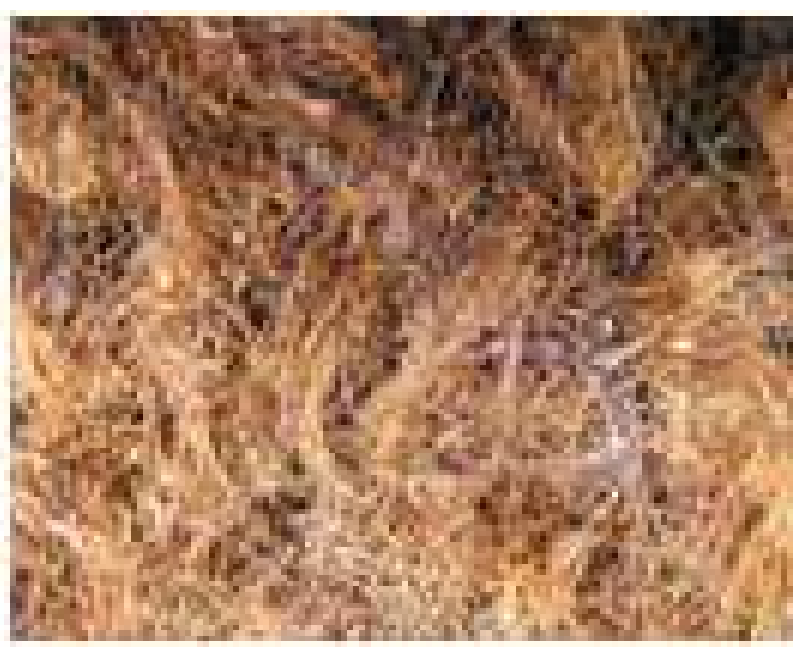

(b)

Fig. 1. (a) fibre size of date palm (b) fibre size of coconut coir

\subsection{Compressing Fibre Process}

The fibre compression process consists of several preparation and processing steps as follows.

\subsubsection{Preparation of the Mould}

Moulds are prepared using lumber material with a thickness of $30 \mathrm{~mm}$. The mould dimensions are $400 \times 400 \times 300 \mathrm{~mm}$ as a high rectangular box and are open on both sides.

\subsubsection{Process Weight}

The fibre filaments were then weighed to the correct mass and densities on the samples. Table 1 shows the mass, densities and thickness required for the samples.

\subsubsection{Process Arrange Model}

Fibre filaments were manually arranged in the mould, layer by layer, using the hand to press on the fibers to make sure that the fibers are evenly distributed over the entire mould, as specified according to mass.

\subsubsection{Manual Pressing}

The compression of fibre layers was performed based on the required thickness gradations. A machine was used for manual compression. The pressure varied with the thickness and weight of the pieces. In addition, the time required was determined according to thickness of the piece.
Table 1. Illustrates the density, thickness and mass of the DPF and CCF for absorption test

\begin{tabular}{llll}
\hline Type of Fiber & Density $\left(\mathrm{kg} / \mathrm{m}^{3}\right)$ & Thickness $(\mathrm{mm})$ & Mass $(\mathrm{g})$ \\
\hline CCF & 130 & 20 & 416 \\
CCF & 159 & 40 & 1017 \\
DPF & 100 & 20 & 320 \\
DPF & 90 & 40 & 576 \\
\hline
\end{tabular}

\subsection{Spraying Process}

In order for the fibers to be formed properly, latex should be used for additional treatment. Latex was sprayed to coat all of the surfaces of the pressed fibers. This treatment maintained the fibers in the same shape.

\subsection{Hot Compress Process}

After finishing spraying the latex, preparation for the final process began with placing the piece on the plate. At the same time, the machine was run for 30 minutes before beginning compression to reach a temperature of $100^{\circ} \mathrm{C}$. The piece was surrounded on both sides with rods at the same height as the thickness required. Then, the sample was covered with the plate. The pressing period was approximately $5 \mathrm{~min}$ and the pressure was set at $170 \mathrm{~kg} / \mathrm{cm}^{2}$.

\subsection{Test}

Allard studied the estimation of the dissipative properties of sound with a rigid wall. Therefore, some physical parameters were added to the model to make 
the model more useful for the treatment of the date palm fibre. For this reason, latex is a very important component of this research. When added to the date palm fibre, all of the surfaces then became one component having a higher porosity with an increase in the stiffness of the fibers, as presented by (Nor et al., 2010a; Ayub et al., 2009).

The experimental setups for the acoustic absorption coefficient and impedance measurements (two microphone-transfer function method) were undertaken in accordance with ISO 10534-2 and ASTM E1050-98. The impedance tube consisted of two steel tubes with a diameter of $100 \mathrm{~mm}$ for low frequency and $28 \mathrm{~mm}$ for high frequency on the SCS9020B/K measurement systems.

The calibration of two $1 / 4$ " microphones of type GRAS-26AK was performed with a noise generator G10 from a loud speaker of type 4206 SCS 9020B/K.

An indicator of frequency functions is the incidence acoustic absorption coefficient, which is between (0-1). This value represents the amount of sound energy being absorbed by the fibre sample. There are several other ways of estimating the absorption coefficients of the materials. The procedures for testing with an impedance tube are shown in Fig. 2 and Fig. 3.

The sample was inserted into the holder to prepare for the test, which began at low frequencies. To translate the sound waves to a digital signal, the SCS 8100 software was used to save the output signal data. The same procedures were repeated for a highfrequency band.

\section{RESULTS}

Test results are presented in Fig. 4-6. These measurements were taken via an impedance tube at a frequency between $87.5-5 \mathrm{kHz}$ for fibers without backing. Acoustic Absorption Coefficients (AACs) will be compared at different thicknesses and densities.

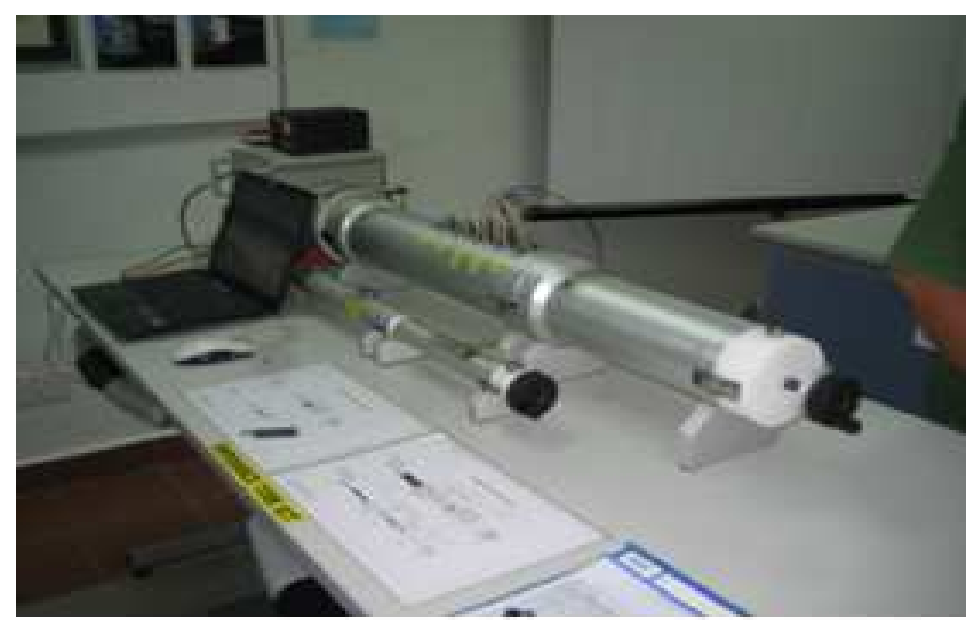

Fig. 2. Impedance tube instrument

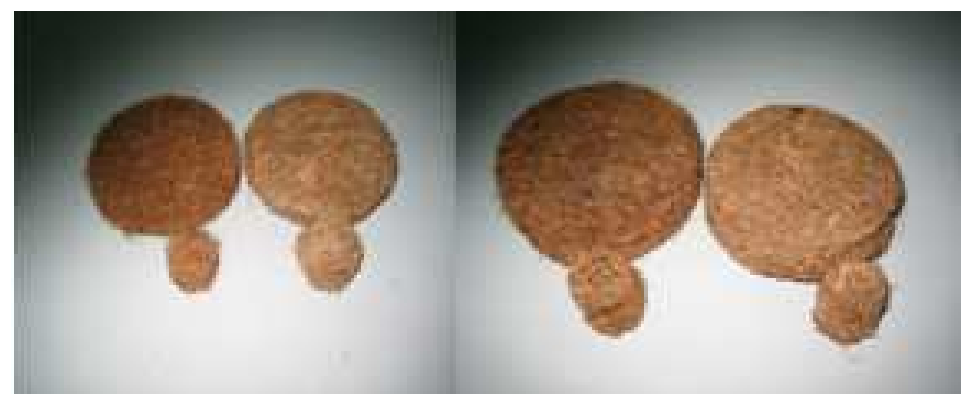

Fig. 3. Fiber samples test of DPF, CCF $20 \mathrm{~mm}$ and $40 \mathrm{~mm}$ thickness with diameter (100 mm, $28 \mathrm{~mm})$ 


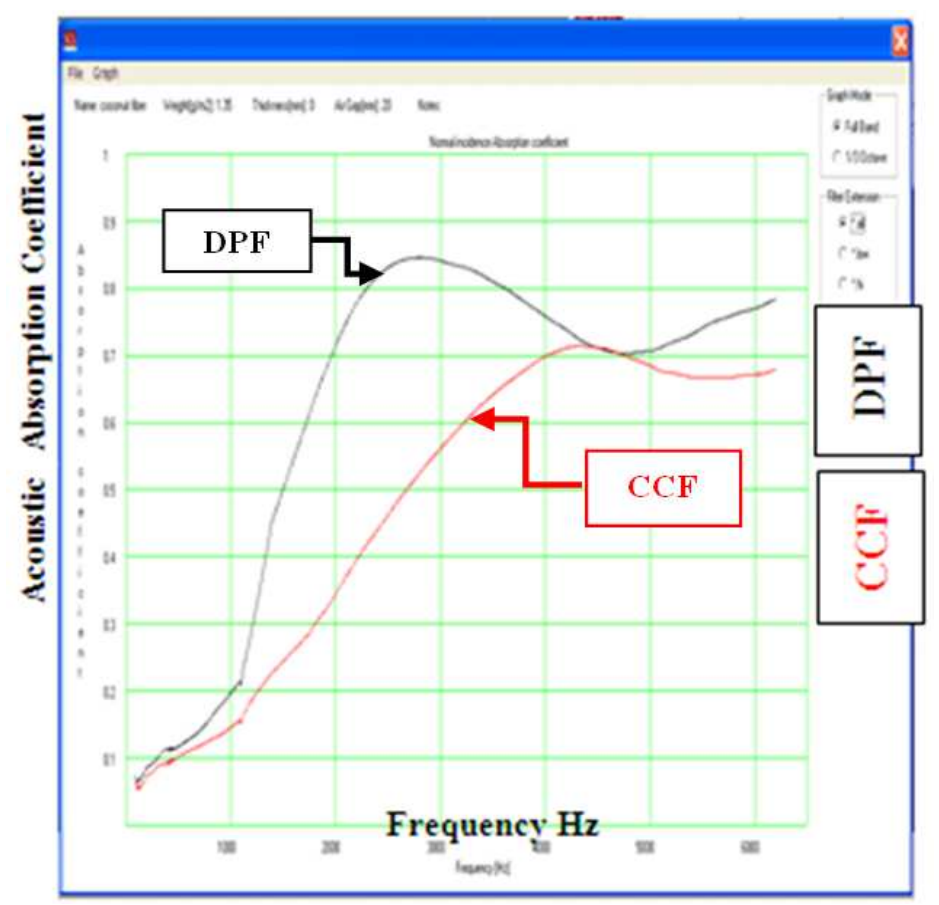

Fig. 4. The comparison between DPF and CCF $20 \mathrm{~mm}$ thickness

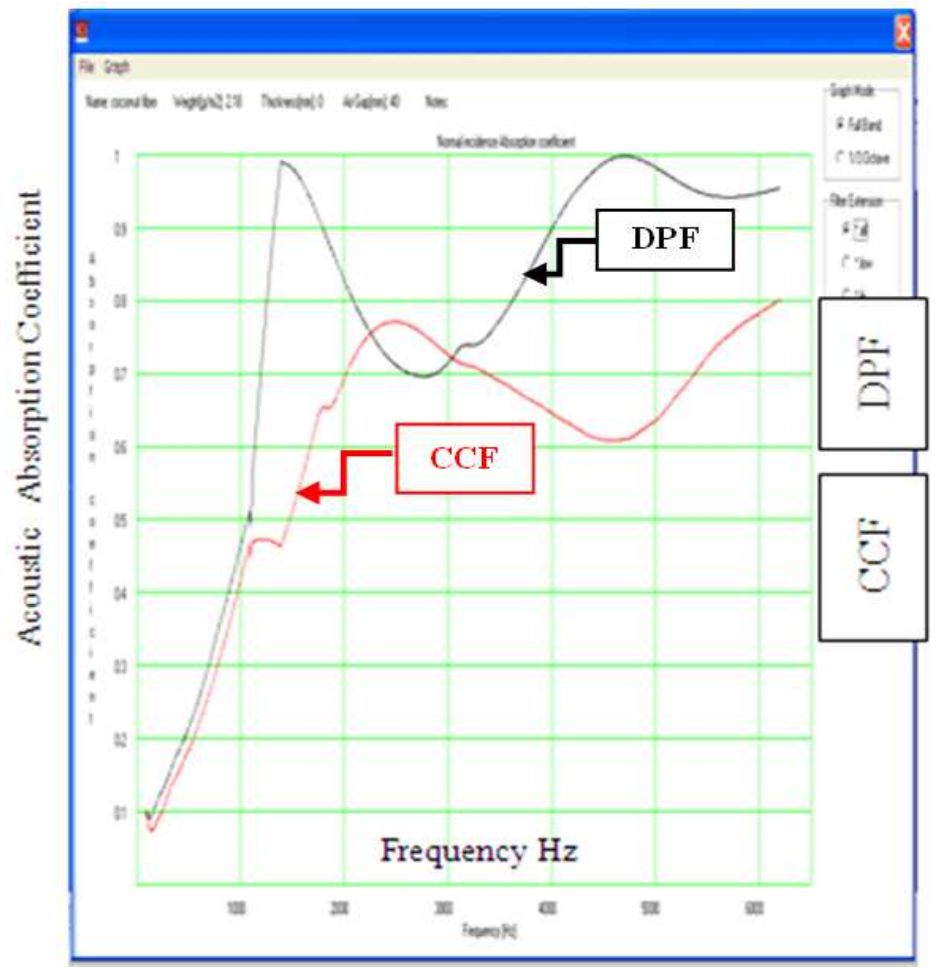

Fig. 5. The comparison between DPF and CCF $40 \mathrm{~mm}$ thickness 


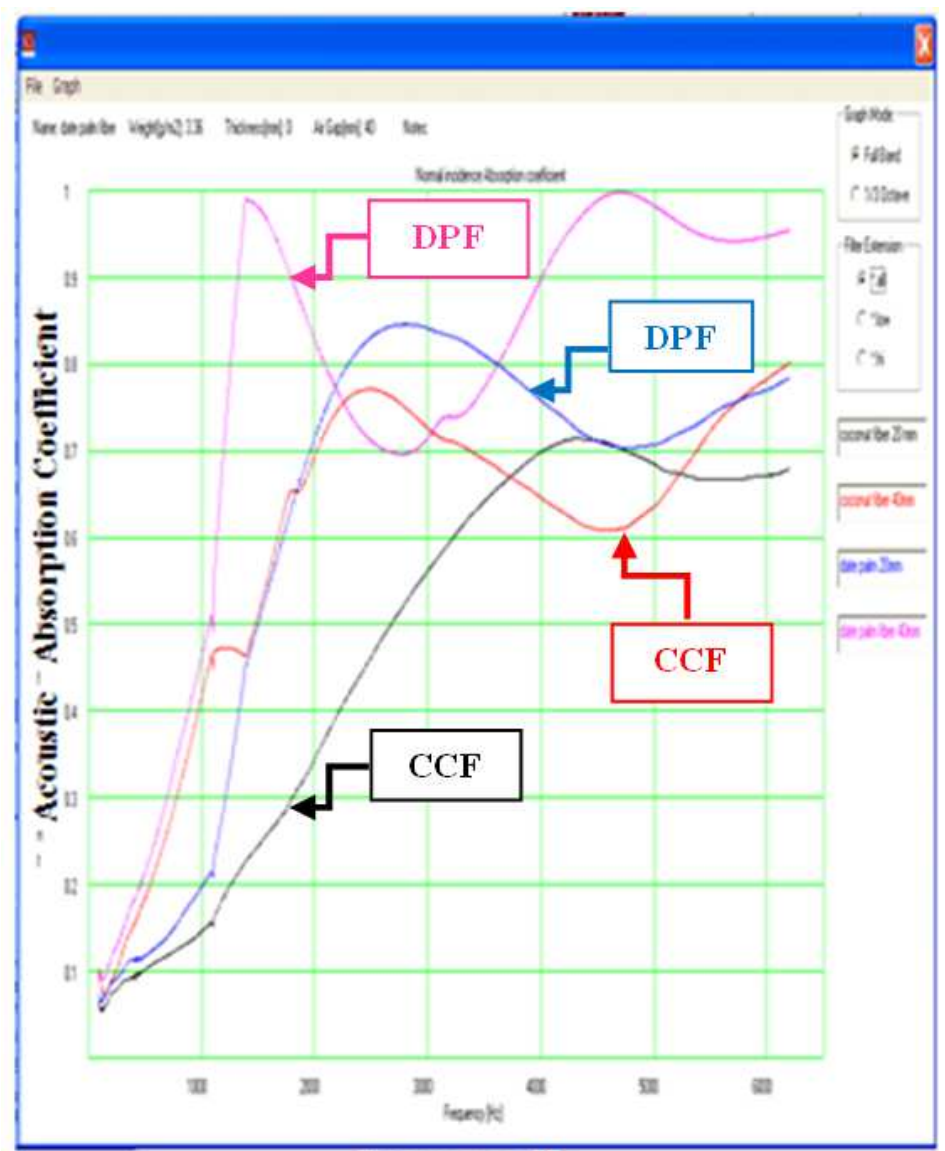

Frequency $\mathrm{Hz}$

Fig. 6. The comparison between samples thicknesses of DPF and CCF $20 \mathrm{~mm}$ and $40 \mathrm{~mm}$

\section{DISCUSSION}

\subsection{Effect of Bulk Density and Thickness}

The absorption coefficients of the $20 \mathrm{~mm}$ and $40 \mathrm{~mm}$ thicknesses of two fibers are shown in Fig. 4 and 5. The results show that increasing the density of the porous material enhances the absorption of the fibre and moves the peaks toward a lower frequency. Table2 illustrates the differences between the densities of the two fibers. For this reason, the effect of the fibre sizes of these materials distinctly promotes acoustic absorption in conjunction with decreasing fibre size (Koizumi et al., 2002; Lee and Joo, 2003) Date palm fibre has a smaller size diameter $123 \mu \mathrm{m}$ than coconut fibre $252 \mu \mathrm{m}$ and this behaviour can be attributed to the changes of two basic substance characteristics: Tortuosity and flow resistivity with change in fibre size. Therefore, flow resistivity is inversely proportional to fibre diameter, while tortuosity indicates the transmission path of sound waves (Cox, 2009).
Table 2. Illustrates the maximum value of acoustic absorption coefficient

\begin{tabular}{llll}
\hline $\begin{array}{l}\text { Type of } \\
\text { Fiber }\end{array}$ & $\begin{array}{l}\text { Thickness } \\
(\mathrm{mm})\end{array}$ & $\begin{array}{l}\text { Maximum } \\
\text { value of AAC }\end{array}$ & $\begin{array}{l}\text { Frequencies } \\
(\mathrm{Hz})\end{array}$ \\
\hline CCF & 20 & 0.71 & $4184.38-4575$ \\
CCF & 40 & 0.77 & $2434.38-2543.75$ \\
DPF & 20 & 0.84 & $2606.25-3025$ \\
DPF & 40 & 0.98 & $1381.25-1506.25$ \\
& & 0.99 & $4521.88-4906.25$ \\
\hline
\end{tabular}

Thinner fibers result in the requirement of less fibre to reach an equal volume density for the same thickness (Nor et al., 2010b). Moreover, thin fibers result in a more tortuous path and higher airflow resistance of porous substances, which promotes the absorption coefficient and shifting to the lower frequencies. In addition, thin fibers can move more easily than thick fibers in a sound wave, which induces vibration in air. These results indicate that fibre size diameter plays an important role 
in the improvement of sound absorption. These fibers showed good performance at low to medium frequencies, but the performance deteriorated at higher frequencies (Hosseini et al., 2010; Rozli et al., 2009b). However, at lower fibre density and smaller diameter, the fibers were able to show good absorption coefficient performance.

\subsection{Physical Effect}

These micro porous materials were mixed with latex, which was used for the physical treatment of these materials. The introduction of latex to the samples adds stiffness, so that sound can be significantly dissipated as it travels through the material (Rozli et al., 2009b). The date palm fibre showed good performance at low and high frequencies, but the performance deteriorated at medium frequencies. However, coconut fibre showed good performance at low to medium frequencies, but the performance deteriorated at high frequency, as shown in Fig. 6. At lower fibre densities and smaller diameters, the fibers show good absorption coefficient performance. Comparison of the effectiveness of a sound absorber depends on the structural architecture of this micro porous material (Hong et al., 2007). Date palm fibre is thinner $123 \mu \mathrm{m}$ than coconut fibre $252 \mu \mathrm{m}$ which means it is able to improve the acoustic absorption coefficient at low and high frequencies (Dias et al., 2007).

\section{CONCLUSION}

In this study, it is shown that date palm fibre and coconut coir fibre have the potential to replace common synthetic fibrous materials such as glass wool, rock wool and asbestos. These results indicate that fibre size plays an important role in the improvement of sound absorption. The effect of the fibre sizes of these micro porous materials distinctly promotes acoustic absorption with decreasing fibre size; date palm fibre has a smaller size fibre $123 \mu \mathrm{m}$ than coconut fibre $252 \mu \mathrm{m}$. Increasing the thickness of the natural fibre layer will increase absorption and move the absorption peak towards lower frequencies. The absorption properties of natural fibers can also be increased by increasing the bulk density of the material. Natural fibers seem to provide better absorption properties because of increased bulk density due to extra matrix material. These results demonstrate that more strategically designed layers and configurations of natural fibers could increase noise mitigation. As a green technology product, these innovative absorption panels may have a bright future because they are cheaper, lighter and more eco-friendly than other common fibrous materials.

\section{REFERENCES}

Arenas, J.P. and M.J. Crocker, 2010. Recent trends in porous sound-absorbing materials. Sound Vibration, 44: $12-18$.

Ayub, M., M.J.M. Nor, N. Amin, R. Zulkifli and M. Hosseini et al., 2009. Analysis on sound absorption of natural coir fiber using delany-bazley model. Proceedings of the International Conference on Mechanical Engineering, Dec. 26-28, Dhaka, Bangladesh, pp: 1-6.

Cox, T.J., 2009. Acoustic absorbers and diffusers: Theory, design and application. Taylor and Francis Group.

Dias, T., R. Monaragala, P. Needham and E. Lay, 2007. Analysis of sound absorption of tuck spacer fabrics to reduce automotive noise. Measurement Sci. Technol., 18: 2657-2657. DOI: 10.1088/0957-0233/18/8/042

Ersoy, S. and H. Kucuk, 2009. Investigation of industrial tea-leaf-fibre waste material for its sound absorption properties. Applied Acoustics, 70: 215-220. DOI: 10.1016/j.apacoust.2007.12.005

Gupta, S. and C. Ghatak, 2011. Environmental noise assessment and its effect on human health in an urban area. Int. J. Environ. SCI., 1: 1954-1964.

Hong, Z., L. Bo, H. Guangsu and H. Jia, 2007. A novel composite sound absorber with recycled rubber particles. J. Sound Vibration, 304: 400-406. DOI: 10.1016/j.jsv.2007.02.024

Hosseini, F.M., M.J.M. Nor, M. Ayub, Z.A. Leman, 2010. Utilization of coir fiber in multilayer acoustic absorption panel. Applied Acoustics, 71: 241-249. DOI: 10.1016/j.apacoust.2009.09.003

Khedari, J., N. Nankongnab, J. Hirunlabh and S. Teekasap, 2004. New low-cost insulation particleboards from mixture of durian peel and coconut coir. Build. Environ., 39: 59-65. DOI: 10.1016/j.buildenv.2003.08.001

Khedari, J., S. Charoenvai and J. Hirunlabh, 2003. New insulating particleboards from durian peel and coconut coir. Build. Environ., 38: 435-441. DOI: 10.1016/S0360-1323(02)00030-6

Koizumi, T., N. Tsujiuchi and A. Adachi, 2002. The development of sound absorbing materials using natural bamboo fibers. High Performance Structure and Composites. 
Lee, Y. and C. Joo, 2003. Sound absorption properties of recycled polyester fibrous assembly absorbers. Autex Res. J., 3: 78-84.

Mahzan, S., A.M.A. Zaidi, N. Arsat, M.N.M. Hatta and R. Mohideen, 2010. Study on sound absorption properties of coconut coir fibre reinforced composite with added recycled rubber. Int. J. Integrated Eng.

Nor, M.J.M., M. Ayub, R. Zulkifli, N. Amin and M.H. Fouladi et al., 2010a. Effect of compression on the acoustic absorption of coir fiber. Am. J. Applied Sci., $\quad 7$ : 1285-1290. DOI: 10.3844/ajassp.2010.1285.1290

Nor, M.J.M., M. Ayub, R. Zulkifli, N. Amin and M.H. Fouladi et al., 2010b. Effect of different factors on the acoustic absorption of coir fiber. J. Applied Sci., 10: 2887-2892. DOI: 10.3923/jas.2010.2887.2892
Suter, A.H., 2002. Construction noise: Exposure, effectsand the potential for remediation; A review and analysis. AIHA J., 63: 768-789. DOI: 10.1202/00028894(2002)063<0768:CNEEAT $>2.0 . C O ; 2$

Yang, H.S., D.J. Kim and H.J. Kim, 2003. Rice strawwood particle composite for sound absorbing wooden construction materials. Bioresource Technol., 86: 117121. DOI: 10.1016/S0960-8524(02)00163-3

Rozli, Z., M. Nor, M. Jailani, I.A. Rasdan and N.M., Zaki, 2009a. Comparison of acoustic properties between coir fibre and oil palm fibre. Eur. J. Sci. Res., 33: 144-144.

Rozli, Z., M. Nor, M. Jailani, I.A. Rasdan and N.M., Zaki, 2009b. Effect of perforated size and air gap thickness on acoustic properties of coir fibre sound absorption panels. Eur. J. Sci. Res., 28: 242-242. 